\title{
Pointwise Estimates for Monotone Polynomial Approximation
}

\author{
Ronald A. DeVore and Xiang Ming Yu
}

\begin{abstract}
We prove that if $f$ is increasing on $[-1,1]$, then for each $n=1,2, \ldots$, there is an increasing algebraic polynomial $P_{n}$ of degree $n$ such that $\mid f(x)-$ $P_{n}(x) \mid \leq c \omega_{2}\left(f, \sqrt{1-x^{2}} / n\right)$, where $\omega_{2}$ is the second-order modulus of smoothness. These results complement the classical pointwise estimates of the same type for unconstrained polynomial approximation. Using these results, we characterize the monotone functions in the generalized Lipschitz spaces through their approximation properties.
\end{abstract}

\section{Introduction}

Several results show that in some sense monotone approximation by algebraic polynomials performs as well as unconstrained approximation. For example, Lorentz and Zeller [6] have shown that for each increasing function $f$ in $C(I)$, $I:=[-1,1]$, there is an increasing polynomial $P_{n}$ of degree $n$ that satisfies

$$
\left\|f-P_{n}\right\| \leq c \omega\left(f, n^{-1}\right), \quad n=1,2, \ldots,
$$

where $\omega$ is the modulus of continuity of $f$ and all norms here and throughout are the uniform norm on $I$.

More generally, for any $k=0,1, \ldots$, there are increasing $P_{n}$ that satisfy

$$
\left\|f-P_{n}\right\| \leq c n^{-k} \omega\left(f^{(k)}, n^{-1}\right), \quad n=1,2, \ldots .
$$

When $k=1$, this is a result of Lorentz [5], whereas the general case was proved by DeVore [3]. The cases $k=0,1$ are much easier to prove than the general case, since they can be proved using linear methods; in contrast, the proof in [3] uses rather involved nonlinear techniques.

It is well-known that for unconstrained approximation, much improvement can be made in estimates of the form (1.1) when $x$ is near an end point of $I$. Such

Date received: September 11, 1984. Date revised: January 24, 1985. Communicated by Edward B. Saff.

1980 AMS (MOS) subject classifications: 41 A 10, 41 A 25, 41 A 29.

Key words and phrases: Monotone approximation by algebraic polynomials, Degree of approximation, Pointwise estimates. 
improvements take the form of replacing $n^{-1}$ in (1.1) with either $\Delta_{n}(x)$ $:=\sqrt{1-x^{2}} / n+1 / n^{2}$ or just $\sqrt{1-x^{2}} / n$. In fact, such improved estimates are needed if we wish to characterize the smoothness of a function by its degree of approximation by algebraic polynomials.

In this article, we are interested in pointwise estimates for monotone approximation. The only result of this type that we know of is by Beatson [1]. He proved that the estimate

$$
\left|f(x)-P_{n}(x)\right| \leq c \omega\left(f, \Delta_{n}(x)\right), \quad x \in I, \quad n=1,2, \ldots
$$

holds for suitable increasing polynomials $P_{n}$ whenever $f$ is increasing. Among other things, we shall show that this can be improved to allow second-order smoothness as measured by the second-order modulus of smoothness $\omega_{2}$.

Theorem 1. If $f$ is increasing on $I$, then for each $n=1,2, \ldots$, there is an increasing polynomial $P_{n}$ of degree $n$ such that

$$
\left|f(x)-P_{n}(x)\right| \leq c \omega_{2}\left(f, \frac{\sqrt{1-x^{2}}}{n}\right), \quad x \in I .
$$

Notice that we have replaced $\Delta_{n}(x)$ with the smaller quantity $\sqrt{1-x^{2}} / n$. For unconstrained approximation, such results were given first by Teljakovskii [7] (for $\omega$ ), and later by DeVore [2] (for $\omega_{2}$ ). From the standard properties of $\omega_{2}$, we see that (1.3) contains the improved form of (1.2) as well as improved estimates of the form (1.1b) when $k=1$.

With Theorem 1 and the classical converse theorems for approximation by algebraic polynomials, we have the following result, which characterizes the Lip* $\alpha$ spaces, which are defined as the set of all $f$ such that $\omega_{2}(f, t)=O\left(t^{\alpha}\right)$, $0<\alpha<2$.

Theorem 2. If $0<\alpha<2$, then a function $f$ is increasing and in $\mathrm{Lip}^{*} \alpha$ if and only if for each $n=1,2, \ldots$, there is an increasing algebraic polynomial $P_{n}$ of degree $n$ such that

$$
\left|f(x)-P_{n}(x)\right| \leq c\left(\frac{\sqrt{1-x^{2}}}{n}\right)^{\alpha}, \quad x \in I
$$

We do not know whether this result holds for $\alpha \geq 2$. This would require at the least a refinement of (1.1b) for $k \geq 2$ in which $1 / n$ is replaced by $\Delta_{n}(x)$.

\section{Proofs}

Our proof is based on a two-stage approximation. We first approximate $f$ by an increasing piecewise linear function $S_{n}$ that approximates to the accuracy of the right side of (1.3). We then approximate $S_{n}$ by an increasing algebraic polynomial. 
$S_{n}$ is constructed as follows. We shall select points $-1=\xi_{-n}<\xi_{-n+1}<\cdots$ $<\xi_{n}=1$ (to be defined precisely later) that are more densely distributed near the end points of $I$. Roughly speaking, these points are uniformly distributed with respect to the Chebyshev measure $d x / \sqrt{1-x^{2}} . S_{n}$ is then the piecewise linear function that interpolates $f$ at the $\xi_{k}, k=-n, \ldots, n$. If we let $s_{j}$ be the slopes

$$
s_{j}:=\frac{f\left(\xi_{j+1}\right)-f\left(\xi_{j}\right)}{\xi_{j+1}-\xi_{j}}, \quad j=-n, \ldots, n-1,
$$

then $S_{n}$ can be represented by the truncated power functions $\Phi_{j}(x):=\left(x-\xi_{j}\right)_{+}$:

$$
s_{n}(x)=f(-1)+s_{-n}(x+1)+\sum_{j=-n+1}^{n-1}\left(s_{j}-s_{j-1}\right) \Phi_{j}(x) .
$$

It is clear that $S_{n}$ is increasing if $f$ is. Also, if $f$ is twice continuously differentiable, $\left\|f^{\prime \prime}\right\| \leq M$, then (Newton's formula)

$$
\left|f(x)-S_{n}(x)\right| \leq(M / 2)\left|\left(x-\xi_{j}\right)\left(x-\xi_{j+1}\right)\right|, \quad \xi_{j} \leq x \leq \xi_{j+1} .
$$

We shall now construct a polynomial approximation to $S_{n}$. To do this, we first construct polynomials $R_{j}, j=-n, \ldots, n-1$, that approximate the truncated powers $\Phi_{j}$. The construction of $R_{j}$ begins with trigonometric polynomials $T_{j}$ that are good approximations to the characteristic functions $\chi_{0}:=0$ and $\chi_{j}:=\chi_{\left[-t_{j}, t_{j}\right]}$, $j=1, \ldots, 2 n$, with $t_{j}:=j \pi / 2 n, j=0,1, \ldots, 2 n$.

Let $K_{n}$ denote the Jackson kernel

$$
K_{n}(t):=a_{n}\left(\frac{\sin n t / 2}{\sin t / 2}\right)^{8} ; \quad \int_{-\pi}^{\pi} K_{n}(t) d t=1 .
$$

We recall that the $K_{n}$ satisfy (see [4, p. 57])

$$
\begin{gathered}
\int_{-\pi}^{\pi}|t|^{j} K_{n}(t) d t \leq c n^{-j}, \quad j=0,1, \ldots, 7, \\
c_{0} n^{-7} \leq a_{n} \leq c_{1} n^{-7} .
\end{gathered}
$$

Here and throughout $c, c_{0}$, and $c_{1}$ denote absolute constants; the value of $c$ may vary with each occurrence, even on the same line. We define

$$
T_{j}(t):=\int_{t-t_{j}}^{t+t_{j}} K_{n}(u) d u, \quad j=0,1, \ldots, 2 n .
$$

In particular, $T_{0}=\chi_{0}$ and $T_{2 n}=\chi_{2 n}$. We define

$$
d_{j}(t):=\max \left(n \operatorname{dist}\left(t,\left\{-t_{j}, t_{j}\right\}\right) ; 1\right) \text {. }
$$

Lemma 3. For $j=0,1, \ldots, 2 n$, we have

$$
\begin{gathered}
\left|\chi_{j}(t)-T_{j}(t)\right| \leq c\left(d_{j}(t)\right)^{-7}, \quad-\pi \leq t \leq \pi \\
\int_{-\pi}^{\pi}\left|\sin t \| \chi_{j}(t)-T_{j}(t)\right| d t \leq c\left(\frac{\sin t_{j}}{n}+\frac{1}{n^{2}}\right), \quad-\pi \leq t \leq \pi
\end{gathered}
$$


Proof. When $j=0$ or $j=2 n$, these inequalities are obvious. For the other values of $j$, we let $a:=\min \left(\left|t-t_{j}\right|,\left|t+t_{j}\right|\right)$. Then

$$
\begin{aligned}
\left|\chi_{j}(t)-T_{j}(t)\right| & =\left|\int_{-\pi}^{\pi}\left(\chi_{j}(t)-\chi_{j}(t-u)\right) K_{n}(u) d u\right| \\
& \leq \int_{|u| \geq a} K_{n}(u) d u \leq \int_{-\pi}^{\pi}\left|\frac{u}{a}\right|^{7} K_{n}(u) d u \leq c(a n)^{-7}
\end{aligned}
$$

because of (2.5). Since we also know that $0 \leq T_{j}(t) \leq 1$, and hence $\mid \chi_{j}(t)-$ $T_{j}(t) \mid \leq 1$, we have (2.7a).

To prove (2.7b) we multiply (2.7a) by $|\sin t|$ and integrate; this leaves us the task of estimating $\Sigma:=\int_{-\pi}^{\pi} \mid \sin t k\left(d_{j}(t)\right)^{-7} d t$. We write

$$
\frac{1}{2} \Sigma=\int_{0}^{\pi}|\sin t|\left(d_{j}(t)\right)^{-7} d t=\int_{I_{1}}+\int_{I_{2}},
$$

with $I_{1}:=\left[t_{j}-1 / 2 n, t_{j}+1 / 2 n\right]$ and $I_{2}:=[0, \pi]-I_{1}$. Since $\left|I_{1}\right| \leq 1 / n$ and $|\sin t| \leq c\left|\sin t_{j}\right|$ for $t \in I_{1}$, we have $\int_{I_{1}} \leq c\left|\sin t_{j}\right| / n$. To estimate the integral over $I_{2}$, we note that $|\sin t| \leq\left|\sin t_{j}\right|+\left|t-t_{j}\right|$, and therefore

$$
\begin{aligned}
\int_{I_{2}} & \leq c \int_{I_{2}}\left(n\left|t-t_{j}\right|\right)^{-7}\left(\left|\sin t_{j}\right|+\left|t-t_{j}\right|\right) d t \\
& \leq c\left(\frac{\left|\sin t_{j}\right|}{n}+\frac{1}{n^{2}}\right) .
\end{aligned}
$$

Now let $r_{j}(x):=T_{n-j}(t), x=\cos t$, and for $x \in[-1,1]$, we define

$$
R_{j}(x):=\int_{-1}^{x} r_{j}(u) d u, \quad j=-n, \ldots, n .
$$

In particular, $R_{-n}(x)=x+1=\Phi_{-n}(x)$ and $R_{n}(x)=0$. The points $\xi_{j}$ are defined by the equations

$$
1-\xi_{j}:=R_{j}(1), \quad j=-n, \ldots, n .
$$

In particular, $\xi_{-n}=-1$ and $\xi_{n}=1$. We now develop the relevant properties of the points $\xi_{j}$ and the polynomials $R_{j}$.

From the definition of the polynomials $T_{j}$, we have $T_{n-j}-T_{n-(j+1)} \geq 0$. Hence, $r_{j}-r_{j+1} \geq 0$, and therefore

$$
R_{j}-R_{j+1} \text { is increasing, } j=-n, \ldots, n-1 \text {. }
$$

This gives that $-1=\xi_{-n}<\xi_{-n+1}<\cdots<\xi_{n}=1$. Further properties of the $\xi_{j}$ are given by

Lemma 4. With $\delta_{j}:=n^{-1}\left(\sin t_{n-j}\right)+n^{-2}$, we have

$$
\begin{aligned}
\left|\xi_{j}-\cos t_{n-j}\right| & \leq c n^{-1} \sin t_{n-j}, \quad j=-n, \ldots, n, \\
\left|\cos ^{-1} \xi_{j}-t_{n-j}\right| & \leq c n^{-1}, \quad j=-n, \ldots, n, \\
c_{0} \delta_{j} \leq \xi_{j+1}-\xi_{j} & \leq c_{1} \delta_{j}, \quad j=-n, \ldots, n-1, \\
c_{0} \delta_{j} \leq \delta_{j+1} & \leq c_{1} \delta_{j}, \quad j=-n, \ldots, n-1 .
\end{aligned}
$$


Proof. We shall use the fact that $\sin t_{k} \geq c \sin t_{k+1}$ and $\sin t_{k} \geq \sin \pi / 2 n \geq 1 / n$, $0<k<2 n$. In particular, this shows that (2.9d) is valid. Now, (2.9a) and (2.9b) are trivially true when $j=-n$ or $j=n$. Hence, we check these only for other values of $j$. From (2.7b) and the definition of the $\xi_{j}$, we have

$$
\left|\xi_{j}-\cos t_{n-j}\right|=\left|\int_{0}^{\pi}\left(\chi_{n-j}(t)-T_{n-j}(t)\right) \sin t d t\right| \leq c \delta_{j} \leq c n^{-1} \sin t_{n-j} .
$$

This gives (2.9a), and writing

$$
\xi_{j+1}-\xi_{j}=\left(\xi_{j+1}-\cos t_{n-j-1}\right)+\left(\cos t_{n-j-1}-\cos t_{n-j}\right)+\left(\cos t_{n-j}-\xi_{j}\right),
$$

it also gives the right inequality in (2.9c).

For the remaining proofs, we consider only $j \geq 0$. Similar arguments prove the case when $j<0$. To prove the left inequality in (2.9c), we let $B=\left[t_{n-j}-\right.$ $\left.\pi / 4 n, t_{n-j}\right]$. Then $\sin t \geq c \sin t_{n-j}$ on $B$, and so

$$
\begin{aligned}
\xi_{j+1}-\xi_{j} & =\int_{0}^{\pi}\left(T_{n-j}(t)-T_{n-j-1}(t)\right) \sin t d t \\
& \geq c|B| \sin t_{n-j} \inf _{B}\left(T_{n-j}-T_{n-j-1}\right) \\
& \geq c n^{-1} \sin t_{n-j} \inf _{B}\left(T_{n-j}-T_{n-j-1}\right) .
\end{aligned}
$$

Now for $t \in B$, we have $[0, \pi / 4 n] \subseteq\left[t-t_{n-j}, t-t_{n-j-1}\right]=: A$. Hence, from the definition (2.6) of the $T_{k}$, we have, for $t \in B$,

$$
T_{n-j}(t)-T_{n-j-1}(t) \geq \int_{A} K_{n}(u) d u \geq \int_{0}^{n / 4 n} K_{n}(u) d u \geq c,
$$

where the very last inequality uses $(2.5 \mathrm{~b})$ and the fact that $(\sin n u / 2) /(\sin u / 2) \geq$ $c n$ for $0 \leq u \leq \pi / 4 n$. Using this in (2.10) proves the left inequality of $(2.9 \mathrm{c})$.

Finally, we prove (2.9b) for $j=0, \ldots, n-1$. Let $J$ be the smallest interval that contains $\cos ^{-1} \xi_{j}$ and $t_{n-j}$. We claim that for $n$ sufficiently large,

$$
\sin t \geq \sin t_{n-j} / 2 \geq c \sin t_{n-j}, \quad \text { for half of the } t \in J .
$$

This is clear if $\cos ^{-1} \xi_{j} \leq t_{n-j}\left((2.11)\right.$ holds on $\left.J \cap\left[t_{n-j} / 2, t_{n-j}\right]\right)$ or if $t_{n-j} \leq$ $\cos ^{-1} \xi_{j} \leq \pi / 2((2.11)$ holds on all of $J)$. On the other hand, if $\cos ^{-1} \xi_{j} \geq \pi / 2$, then from (2.9a), $\left|\xi_{j}-\cos t_{n-j}\right| \leq c / n$, and hence $t_{n-j} \geq \pi / 4$ and $\cos ^{-1} \xi_{j} \leq 3 \pi / 4$ provided $n$ is sufficiently large. Therefore (2.11) holds in this case as well. Now integrating (2.11) over $J$ and using (2.9a) gives

$$
c \sin t_{n-j}|J| \leq\left|\xi_{j}-\cos t_{n-j}\right| \leq c n^{-1} \sin t_{n-j} .
$$

This gives $|J| \leq c / n$ provided $n$ is sufficiently large, and (2.9b) follows.

Lemma 5. For $j=-n, \ldots, n-1, x=\cos t$ with $0 \leq t \leq \pi$, we have

$$
\begin{aligned}
& \left|\Phi_{j}(x)-R_{j}(x)\right| \leq c \frac{\sin t}{n}\left(d_{n-j}(t)\right)^{-5}, \\
& \left|\Phi_{j}^{\prime}(x)-R_{j}^{\prime}(x)\right| \leq c\left(d_{n-j}(t)\right)^{-7} .
\end{aligned}
$$


Proof. The case $j=-n$ follows from the fact that $R_{-n}=\Phi_{-n}$. Therefore, we assume $j>-n$. We first prove (2.12b). Since $R_{j}^{\prime}(x)=r_{j}(x)=T_{n-j}(t)$, we have, from $(2.7 a)$,

$$
\begin{aligned}
\left|\Phi_{j}^{\prime}(x)-R_{j}^{\prime}(x)\right| & \leq\left|\chi_{n-j}(t)-T_{n-j}(t)\right|+\chi_{J}(t) \\
& \leq c\left(d_{n-j}(t)\right)^{-7}+\chi_{J}(t),
\end{aligned}
$$

where $J$ is the smallest interval that contains $t_{n-j}$ and $\cos ^{-1} \xi_{j}$. It follows from (2.9b) that $|J| \leq c n^{-1}$. Hence $d_{n-j}(t) \leq c$ for $t \in J$. This means that the second term on the right side of (2.13) is smaller than the first term, and therefore (2.12b) holds.

To prove (2.12a) we shall use the fact that for $k=0,1$ and all $j$,

$$
\begin{array}{ll}
\int_{0}^{t}\left|u-t_{j}\right|^{k}\left(d_{j}(u)\right)^{-7} d u \leq c n^{-k-1}\left(d_{j}(t)\right)^{-5}, & 0 \leq t \leq t_{j}, \\
\int_{t}^{\pi}\left|u-t_{j}\right|^{k}\left(d_{j}(u)\right)^{-7} d u \leq c n^{-k-1}\left(d_{j}(t)\right)^{-5}, & t_{j} \leq t \leq \pi .
\end{array}
$$

For example, the first inequality is proved by writing the integral as a sum of two integrals, the first over $I_{1}:=[0, t] \cap\left[t_{j}-1 / n, t_{j}\right]$ and the second over $I_{2}:=[0, t]$ $\backslash I_{1}$. Then

$$
\begin{aligned}
\int_{I_{2}} & \leq n^{-7} \int_{I_{2}}\left|u-t_{j}\right|^{-7+k} d u \leq c n^{-7} \min \left(n^{6-k},\left|t-t_{j}\right|^{-6+k}\right) \\
& \leq c n^{-k-1}\left(d_{j}(t)\right)^{-5}
\end{aligned}
$$

where we have used the fact that $d_{j}(u) \geq 1$ for all $u$. If the integral over $I_{1}$ is not zero, then $\left|I_{1}\right| \leq 1 / n$ and $d_{j}(t)=1$. Hence,

$$
\int_{I_{1}} \leq n^{-k-1} \leq n^{-k-1}\left(d_{j}(t)\right)^{-5}
$$

as desired. The second inequality in (2.14) is proved in the same way.

Now to prove (2.12a), we write

$$
\begin{aligned}
\Phi_{j}(x)-R_{j}(x) & =\int_{-1}^{x}\left(\Phi_{j}^{\prime}(y)-R_{j}^{\prime}(y)\right) d y \\
& =-\int_{x}^{1}\left(\Phi_{j}^{\prime}(y)-R_{j}^{\prime}(y)\right) d y .
\end{aligned}
$$

If $t \geq t_{n-j}$, we use the first representation in (2.15) and (2.12b) to find that

$$
\left|\Phi_{j}(x)-R_{j}(x)\right| \leq \int_{-1}^{x}\left|\Phi_{j}^{\prime}(y)-R_{j}^{\prime}(y)\right| d y \leq c \int_{t}^{\pi} \sin u\left(d_{n-j}(u)\right)^{-7} d u .
$$

If $\pi / 2 \leq t$, then $\sin u \leq \sin t$ on the interval of integration, and therefore by using (2.14b) with $k=0$, we get (2.12a).

On the other hand, if $t \leq \pi / 2$, then we use $\sin u \leq \sin t+|u-t| \leq \sin t+\mid u$ $-t_{n-j} \mid$ on the interval of integration. Putting this in the right integral of (2.16) 
and using $(2.14 \mathrm{~b})$ gives

$$
\left|\Phi_{j}(x)-R_{j}(x)\right| \leq c \frac{\sin t}{n}\left(d_{n-j}(t)\right)^{-5}+n^{-2}\left(d_{n-j}(t)\right)^{-5} .
$$

But $\sin t \geq n^{-1}$, because $t \geq t_{1}=\pi / 2 n$, and therefore (2.12a) follows in this case.

If $t \leq t_{n-j}$, we use the second representation in (2.15) together with (2.14a) to arrive at the same conclusion.

If $f \in C(I)$, we define

$$
L_{n}(f):=f(-1)+s_{-n} R_{-n}+\sum_{-n+1}^{n-1}\left(s_{j}-s_{j-1}\right) R_{j}
$$

with $s_{j}$ defined by (2.1). If $f$ is increasing, then $s_{j} \geq 0, j=-n, \ldots, n-1$, and since we can also write

$$
L_{n}(f)=f(-1)+\sum_{-n}^{n-1} s_{j}\left(R_{j}-R_{j+1}\right),
$$

it follows from (2.8) that $L_{n}(f)$ is increasing. Also, since $R_{j}(-1)=0$ and $R_{j}(1)=1-\xi_{j}=\Phi_{j}(1)$ for all $j$, we have $L_{n}(f, \pm 1)=f( \pm 1)$.

Theorem 6. If $f^{\prime}$ is absolutely continuous and $\left|f^{\prime \prime}\right| \leq M$ a.e. on $I$, then for each $n=1,2, \ldots$ and each $x \in I$, we have

$$
\left|f(x)-L_{n}(f, x)\right| \leq c M\left(\frac{\sqrt{1-x^{2}}}{n}\right)^{2}
$$

Proof. We will check (2.19) for $x \geq 0$; the case $x<0$ is proved similarly. If $\xi_{j} \leq x \leq \xi_{j+1}$ and $j \leq n-2$ (that is, excluding the rightmost interval), then writing $\theta_{j}:=\cos ^{-1} \xi_{j}$, we have $\left|\theta_{j+1}-t_{n-j-1}\right| \leq c / n$ and hence (2.9) gives

$$
\begin{aligned}
\xi_{j+1}-\xi_{j} & \leq c \delta_{j} \leq c \delta_{j+1}=c\left(\frac{\sin t_{n-j-1}}{n}+\frac{1}{n^{2}}\right) \leq c\left(\frac{\sin \theta_{j+1}}{n}+\frac{1}{n^{2}}\right) \\
& \leq c\left(\frac{\sin t}{n}+\frac{1}{n^{2}}\right) \leq c \frac{\sin t}{n}=c \frac{\sqrt{1-x^{2}}}{n} .
\end{aligned}
$$

Hence,

$$
\begin{aligned}
\left|f(x)-S_{n}(x)\right| & \leq(M / 8)\left(\xi_{j+1}-\xi_{j}\right)^{2} \\
& \leq c M\left(\frac{\sqrt{1-x^{2}}}{n}\right)^{2}, \quad \xi_{j} \leq x \leq \xi_{j+1} .
\end{aligned}
$$

This inequality also holds when $j=n-1$, because $\left(x-\xi_{n-1}\right)(1-x) \leq$ $c \delta_{n-1}(1-x) \leq c(1-x) / n^{2}$ on this interval. Hence, we have

$$
\left|f(x)-S_{n}(x)\right| \leq c M\left(\frac{\sqrt{1-x^{2}}}{n}\right)^{2}, \quad 0 \leq x \leq 1 \text {. }
$$


We now estimate

$$
E(x):=S_{n}(x)-L_{n}(f, x)=\sum\left(s_{j}-s_{j-1}\right)\left(\Phi_{j}(x)-R_{j}(x)\right) .
$$

Here and in what follows, an unsubscripted " $\Sigma$ " denotes the sum for $j=-n+1$ to $j=n-1$. Now $\left|s_{j}-s_{j-1}\right| \leq c M\left(\xi_{j+1}-\xi_{j}\right) \leq c M \delta_{j}$ for each $j$ [see (2.9c)]. Using this and (2.12a) with $x=\cos t$ gives

$$
|E(x)| \leq c M \frac{\sin t}{n} \sum \delta_{j}\left(d_{n-j}(t)\right)^{-5} .
$$

Now for $|j|<n, \quad \delta_{j} \leq n^{-1}\left(\sin t+\left|t-t_{n-j}\right|\right)+n^{-2} \leq c n^{-1}\left(\sin t+\left|t-t_{n-j}\right|\right)$. Hence,

$$
\begin{aligned}
|E(x)| & \leq c M \frac{\sin t}{n^{2}} \sum\left(\sin t+\left|t-t_{n-j}\right|\right)\left(d_{n-j}(t)\right)^{-5} \\
& \leq c M \frac{\sin t}{n^{2}}(\sin t+1 / n) \leq c M\left(\Delta_{n}(x)\right)^{2}, \quad 0 \leq x \leq 1,
\end{aligned}
$$

where we have used the easily verified inequalities

$$
\sum_{j=1}^{2 n-1}\left|t-t_{j}\right|^{k}\left(d_{j}(t)\right)^{-5} \leq c n^{-k}, \quad k=0,1 .
$$

We can improve (2.23) near the end point 1. Differentiating (2.22) and using (2.12b) gives

$$
\left|E^{\prime}(x)\right| \leq c M \sum \delta_{j}\left(d_{n-j}(t)\right)^{-7} \leq c M \Delta_{n}(x), \quad x \in I,
$$

where the sum has been estimated as in (2.23). Integrating this inequality from $x$ to 1 and using the fact that $E(1)=0$ gives

$$
|E(x)| \leq c M\left(1-x^{2}\right) \Delta_{n}(x) .
$$

If we superimpose the two inequalities (2.23) and (2.25), we get (2.19). That is, when $1-x^{2} \geq n^{-2},(2.23)$ gives the desired estimate, and when $1-x^{2} \leq n^{-2}$, (2.25) gives the desired estimate $\left(\Delta_{n}(x) \leq 2 n^{-2}\right.$ in this case).

Proof of Theorem 1. We first prove that the $L_{n}$ are uniformly bounded on $C(I)$. From (2.12), it follows that

$$
\left|\Phi_{j}(x)-R_{j}(x)\right| \leq c n^{-1}\left(\sin t_{n-j}+\left|t-t_{n-j}\right|\right)\left(d_{n-j}(t)\right)^{-5} .
$$

Now from (2.9), $\left|s_{j}\right| \leq 2\|f\| /\left(\xi_{j+1}-\xi_{j}\right) \leq c\|f\| / \delta_{j} \leq c\|f\| / \delta_{j+1}$. Using this with (2.26) in (2.22) gives

$$
\begin{aligned}
\left|L_{n}(f, x)\right| & \leq\left|S_{n}(x)\right|+\sum_{-n+1}^{n-1}\left|s_{j}-s_{j-1}\right|\left|R_{j}(x)-\Phi_{j}(x)\right| \\
& \leq\|f\|+c\|f\| \sum_{-n+1}^{n-1} n^{-1}\left(\sin t_{n-j}+\left|t-t_{n-j}\right|\right)\left(d_{n-j}(t)\right)^{-5} \delta_{j}^{-1} \\
& \leq\|f\|+c\|f\| \sum_{-n+1}^{n-1}\left(1+n\left|t-t_{n-j}\right|\right)\left(d_{n-j}(t)\right)^{-5} \\
& \leq c\|f\|,
\end{aligned}
$$


where the last inequality uses (2.24). This shows that the $L_{n}$ are uniformly bounded.

It is well-known (see, e.g., [2]) that the $K$-functional

$$
K_{2}(f, u):=\inf _{g}\left(\|f-g\|+u^{2}\left\|g^{\prime \prime}\right\|_{\infty}\right)
$$

is equivalent to $\omega_{2}(f, u)$ when $f$ is in $C(I)$; in particular,

$$
K_{2}(f, u) \leq c \omega_{2}(f, u), \quad u>0 .
$$

Given $x \in I$, we fix $x$ and we take $u:=\sqrt{1-x^{2}} / n$. Then from (2.27), there is a $g$ that satisfies

$$
\|f-g\| \leq c \omega_{2}(f, u)
$$

and

$$
u^{2}\left\|g^{\prime \prime}\right\|_{\infty} \leq c \omega_{2}(f, u) .
$$

From Theorem 6 and the linearity of $L_{n}$, we have

$$
\begin{aligned}
\left|f(x)-L_{n}(f, x)\right| & \leq|f(x)-g(x)|+\left|g(x)-L_{n}(g, x)\right|+\left|L_{n}(f-g, x)\right| \\
& \leq\left(1+\left\|L_{n}\right\|\right)\|f-g\|+c\left\|g^{\prime \prime}\right\|_{\infty} u^{2} \\
& \leq c \omega_{2}(f, u)=c \omega_{2}\left(f, \frac{\sqrt{1-x^{2}}}{n}\right) .
\end{aligned}
$$

Since $L_{n}(f)$ is increasing and a polynomial of degree $\leq 8 n$, we have proved Theorem 1.

Acknowledgments. R.A.D. was supported by National Science Foundation Grant DMS 8320562. This work was done while the second author was visiting the University of South Carolina.

\section{References}

1. R. Beatson (preprint): Shape preserving convolution operators.

2. R. DeVore (1976): Degree of approximation. In: Approximation Theory II (G. G. Lorentz, C. K. Chui, L. Schumaker, eds.). New York: Academic Press, pp. 117-162.

3. R. DeVore (1977): Monotone approximation by polynomials. SIAM J. Math. Anal., 8:906-921.

4. G. G. Lorentz (1966): Approximation of Functions. New York: Holt.

5. G. G. Lorentz (1972): Monotone approximation. In: Inequalities III (O. Shisha, ed.). New York: Academic Press, pp. 201-215.

6. G. G. Lorentz, K. Zeller (1969): Degree of approximation by monotone polynomials, II. J. Approx. Theory, 2:265-269.

7. S. Teljakovskii (1966): Two theorems on the approximation of functions by algebraic polynomials. Mat. Sbornik, 70(112):252-265.

R. A. DeVore

Department of Mathematics and Statistics

University of South Carolina

Columbia, South Carolina 29208
X. M. Yu

Department of Mathematics

Nanjing Normal University

Nanjing, People's Republic of China 